\title{
Die nekrotisierende Enterokolitis des Frühgeborenen
}

Florian Guthmann

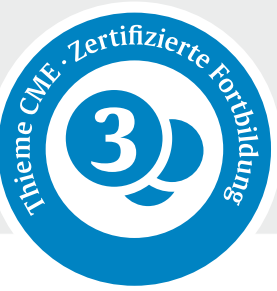

\author{
Die nekrotisierende Enterokolitis (NEK) ist in den USA nach Angaben des Neonatal \\ Research Network die zweithäufigste Todesursache Frühgeborener in der 22. \\ bis vollendeten 28. SSW. Die Ätiologie der NEK wird trotz jahrzehntelanger For- \\ schung noch immer nicht ausreichend verstanden, und die Diagnosestellung \\ einer NEK ist nicht selten problematisch. Schwerpunkte dieses Beitrags sind \\ praktische Aspekte der Prävention und Diagnostik der NEK.
}

\section{Einleitung}

Die nekrotisierende Enterokolitis ist einer der schwerwiegendsten und vielerorts auch der häufigste gastrointestinale Notfall bei Frühgeborenen. Sie ist gefürchtet, weil sie oftmals völlig überraschend auftritt, im Anfangsstadium schwer zu diagnostizieren ist und keine befriedigende Therapie zur Verfügung steht. Die Letalität der NEK ist hoch. Da sehr unreife Frühgeborene inzwischen häufiger überleben, hat die Bedeutung der NEK im Vergleich zu pulmonalen, infektiologischen oder zerebralen Todesursachen zugenommen. Die NEK ist bei Frühgeborenen vor der 29. SSW die häufigste Todesursache im Alter von 8-120 Tagen [1]. Überlebende Kinder können von erheblicher Komorbidität betroffen sein, die entwicklungsneurologische Defizite, Darmpassagestörungen sowie das Kurzdarmsyndrom und entsprechende Komplikationen umfasst.

Die vermutlich erste Beschreibung einer NEK geht auf den Kinderarzt Charles Billard (1800-1832) zurück. Billard hatte Symptome bei einem Findelkind beschrieben, die denen einer NEK sehr ähnlich sind. Bei der Leichenöffnung war die Darmschleimhaut „so erweicht, dass sie mehr einem Brei als einer Membran glich“. Heinrich Willi hat 1944 in Zürich 62 Säuglinge mit einer „bösartigen Enteritis“ beschrieben, von denen zwei Drittel unter $2500 \mathrm{~g}$ wogen. Die Enteritis trat in Clustern auf und war mit Überbelegung der Station assoziiert.

\section{Epidemiologie}

Die NEK ist ganz überwiegend eine Erkrankung des Frühgeborenen ohne Geschlechterwendigkeit. Etwa 5-7\% der Frühgeborenen mit einem Geburtsgewicht von 500-1500g erleiden eine NEK, $85 \%$ der NEKPatienten sind Frühgeborene. Der Zusammenhang der
NEK-Inzidenz mit niedrigem Geburtsgewicht ist an sehr großen Kohorten gezeigt worden, sie variiert jedoch sehr stark: Eine niedrigere Inzidenz findet sich in Japan, der Schweiz und Österreich, eine höhere in Nordamerika, England und Irland [2]. Die Ursache dieser Unterschiede ist unklar.

Die NEK-Inzidenz variiert auch von Zentrum zu Zentrum innerhalb eines Gesundheitssystems. In den am Canadian Neonatal Network teilnehmenden 24 Zentren variiert das Risiko, eine NEK zu erleiden, in den Kliniken mit niedrigster und höchster NEK-Inzidenz auch nach Risikoadjustierung um das Sechsfache [3]. Neben den geografischen Unterschieden der Inzidenz wurden auch zeitliche Häufungen, also NEK-Cluster nach NEKfreien Jahren, beschrieben.

Die NEK mündet nicht selten im septischen Schock und/oder Multiorganversagen mit einer Letalität von $10-50 \%$, wobei Jungen von einer höheren Letalität betroffen sind. Operierte Kinder repräsentieren die schwersten Krankheitsverläufe und weisen wahrscheinlich deshalb die höchste Letalität auf.

\section{Mögliche Folgen}

\section{Kurzdarmsyndrom}

Unmittelbare Folge einer NEK, insbesondere nach einer NEK-OP mit umfangreicher Darmresektion, kann ein Kurzdarmsyndrom sein. Nach Abklingen der akuten Krankheitsphase regeneriert sich die Mukosa und wird ggf. hyperplastisch, funktionelle Reserven, vor allem im Ileum, werden für die Resorptionsleistung des Darmes ausgeschöpft, der Darm adaptiert sich. Es kann aber auch ein Circulus vitiosus entstehen, der über Monate und Jahre eine parenterale Ernährung erfordert. Wichtige prognostische Faktoren für die Dauer der pa- 
- Tab. 1 Dauer (Halbwertszeit) der parenteralen Ernährung bei Kindern mit Kurzdarmsyndrom [4].

\begin{tabular}{|l|l|l|}
\hline $\begin{array}{l}\text { Parenterale Ernäh- } \\
\text { rung (Monate) }\end{array}$ & $\begin{array}{l}\text { Dünndarm- } \\
\text { länge } \mathbf{( c m )}\end{array}$ & $\begin{array}{l}\text { lleozäkalklappe } \\
\text { vorhanden }\end{array}$ \\
\hline$>48$ & $<40$ & nein \\
\hline 31 & $<40$ & ja \\
\hline 22 & $>40$ & nein \\
\hline 10 & $>40$ & ja \\
\hline
\end{tabular}

renteralen Ernährung nach Darmresektion sind die Länge des verbliebenen Dünndarms und das Vorhandensein der lleozäkalklappe ( Tab.1) [4]. Schwerwiegende Komplikationen der parenteralen Ernährung sind eine Cholestase und ein Leberversagen, die durch wiederholte ZVK-assoziierte Septitiden und Nahrungskarenz begünstigt werden. Die Letalität des Kurzdarmsyndroms bei einer Dünndarmlänge von unter $40 \mathrm{~cm}$ beträgt über $25 \%[5,6]$.

\section{Darmpassagestörungen}

Nach einer NEK entstehen bei jedem vierten Kind Darmpassagestörungen, die als narbige Strikturen Folgen der hypoxisch-ischämischen Schädigung einzelner Darmareale, oder als Briden Folgen einer Laparotomie sein können. Beides kann zu erheblich beeinträchtigter Lebensqualität durch chronische lleuszustände führen.

\section{Beeinträchtigung der neurologischen Entwicklung}

Eine langfristige systemische Folge der NEK ist die schwere Beeinträchtigung der neurologischen Entwicklung. Man geht davon aus, dass fast die Hälfte der Frühgeborenen, die eine NEK erlitten haben, von schweren kognitiven und psychomotorischen Beeinträchtigungen betroffen ist [7]. Die vorhandenen Studien sind Kohorten- oder Fallkontrollstudien und unterscheiden sich in ihrem Design und den Einschlusskriterien. Obwohl sich der Einfluss wichtiger Störfaktoren wie Frühgeburtlichkeit, IUGR oder Sepsis in einer Metaanalyse nicht quantifizieren lässt, vermitteln die von Rees und Mitarbeitern analysierten Daten, trotz aller methodischer Limitationen, einen Eindruck von dem Ausmaß der durch die NEK verursachten Spätschäden ( $\mathrm{Tab} .2$ ).

Im Vergleich zur konservativen Therapie haben Kinder, die wegen einer NEK operiert werden mussten, ein zusätzliches Risiko für eine entwicklungsneurologische Störung. Sehr wahrscheinlich ist dieses Risiko nicht durch die Operation begründet, sondern reflektiert besonders schwere Verläufe mit Pneumoperitoneum.

\section{Pathogenese}

Die Pathogenese der NEK ist nicht ausreichend verstanden und multifaktoriell bedingt. Die Kenntnis von Risikofaktoren der NEK, Experimente mit NEK-Tiermodellen und jüngst auch genetische Analysen von Frühgeborenen mit NEK haben unser Verständnis vertieft und zu zahlreichen Hypothesen geführt.

\section{Unreife}

Unreife ist der am längsten bekannte und vermutlich wichtigste Faktor der Pathogenese der NEK. Allerdings beschreibt dieser Begriff eher die betroffene Population, als dass er irgendeine Vorstellung von der Pathogenese der NEK widerspiegelt. In den letzten Jahren wurden Besonderheiten der Entwicklung extrem unreifer Frühgeborener im Vergleich zu Reifgeborenen oder Erwachsenen beschrieben, die eine Vorstellung vermitteln, welches die Substrate der „Unreife“ sein könnten:

- Unreife humane Enterozyten reagieren im Gegensatz zu adulten Enterozyten mit einer exzessiven proinflammatorischen Zytokinproduktion nach Stimulation durch Lipopolysaccharid oder IL-1 $\beta$.

- Neugeborene Ratten reagieren mit der Expression von Hämoxygenase auf hypoxischen Stress 48 Stunden später als juvenile Tiere. Hämoxygenase katalysiert die Synthese von Kohlenmonoxid, das vasodilatatorisch und perfusionssteigernd wirkt.

- Tab.2 Entwicklungsneurologische Folgen der NEK [7].

\begin{tabular}{|c|c|c|c|c|c|c|}
\hline \multirow[t]{2}{*}{ Outcome } & \multicolumn{2}{|l|}{ NEK } & \multicolumn{2}{|c|}{ Keine NEK } & \multirow[t]{2}{*}{ OR } & \multirow[t]{2}{*}{$95 \% \mathrm{Cl}$} \\
\hline & ja (n) & nein (n) & ja (n) & nein (n) & & \\
\hline Zerebralparese & 79 & 393 & 590 & 3984 & 1,55 & $1,19-2,03$ \\
\hline schwere Sehstörung & 10 & 296 & 36 & 2857 & 2,31 & $1,04-5,11$ \\
\hline $\mathrm{MDI}<70$ & 133 & 369 & 882 & 3680 & 1,72 & $1,35-2,19$ \\
\hline $\mathrm{PDI}<70$ & 115 & 328 & 835 & 3605 & 1,71 & $1,34-2,18$ \\
\hline $\mathrm{NDI}<70$ & 166 & 371 & 1243 & 3531 & 1,58 & $1,25-1,99$ \\
\hline
\end{tabular}


- Zusammensetzung und Viskosität des Mukus, der schützenden Schicht zwischen Mukosa und Stuhl, entsprechen erst nach etwa 27 SSW annähernd der von reifen Neugeborenen.

\section{Infektion und Mikrobiom}

Die NEK ist, zumindest im Anfangsstadium, nicht notwendigerweise eine systemische Infektion. Weniger als $15 \%$ der Blutkulturen von Frühgeborenen mit NEK sind positiv. Die am häufigsten nachgewiesenen Erreger sind Enterobacteriaceae, Staphylokokken, Klostridien, Keime der Hautflora und seltener Viren und Pilze. Es scheint keinen NEK-spezifischen Erreger zu geben. Vermutlich ist eine positive Blutkultur Folge einer fortgeschrittenen NEK mit ausgeprägtem „gut barrier failure“ und bakterieller Durchwanderung der Darmwand.

Wesentlich komplexer scheint die Rolle des Stuhlmikrobioms zu sein, welches in den letzten Jahren mit molekularbiologischen Methoden genauer untersucht werden konnte, was mit der konventionellen Stuhlkultur nicht möglich ist. Die Entwicklung der physiologischen Darmflora Neugeborener wird nicht nur durch Antibiotika, sondern auch durch Formulanahrung, Kaiserschnitt oder die bloße Hospitalisierung gestört. Nicht selten sind Frühgeborene allen vier Risikofaktoren ausgesetzt.

\section{Genetik}

Die bis heute bekannten Risikofaktoren für das Auftreten einer NEK erklären weder die Variabilität des Krankheitsverlaufs noch das sporadische oder „zufällige“ Auftreten der NEK. In den letzten Jahren sind Kandidatengene untersucht worden, die eine Rolle bei der intestinalen Immunität, Inflammation oder bei der Regulation des oxidativen Stresses spielen. Meist handelt es sich bei den Untersuchungen um Fragen zur Bedeutung von Einzelnukleotidpolymorphismen (SNP). Da die Untersuchungen auf kleinen Fallzahlen beruhen, sind die Ergebnisse als vorläufig einzustufen. Beispielhaft seien die Gene NFKB und NFKB1A genannt, deren veränderte Expression das NEK-Risiko beeinflusst.

\section{Risikofaktoren und Prävention}

Von den zahlreichen bis heute nachgewiesenen Risikofaktoren lassen sich geringes Geburtsgewicht, Hypotrophie, vorzeitiger Blasensprung, Frühgeburtlichkeit und ethnische Herkunft nicht beeinflussen. Zu den Faktoren, die theoretisch beeinflusst werden können, zählen Beatmung, Sepsis, Geburt außerhalb eines Perinatalzentrums und die arterielle Hypotension, die mit Katecholaminen behandelt werden muss. In zwei Studien schien eine Geburt mittels Kaiserschnitt das Risiko für eine NEK zu senken [8].

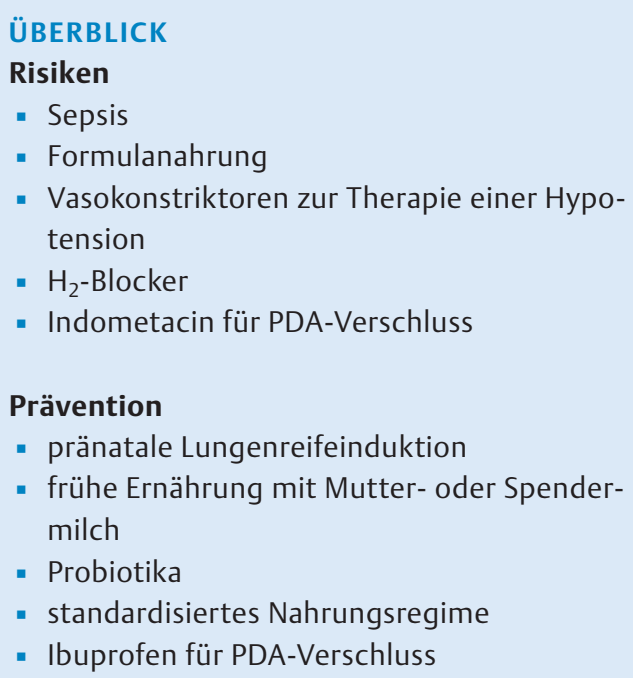

\section{Lungenreife-Induktion}

Die pränatale Lungenreife-Induktion senkt die NEKInzidenz um die Hälfte $(\mathrm{n}=4702$; $\mathrm{RR}=0,50 ; 95 \% \mathrm{Cl}=$ $0,32-0,78)[9]$.

\section{Humane Milch}

Die Vorteile der Ernährung Neugeborener mit Muttermilch sind bekannt: Dies sind ein niedrigerer $\mathrm{pH}$ im Magen, eine gesteigerte Darmmotilität und eine verminderte Permeabilität des Darmepithels, um nur einige zu nennen. Es gibt aber wenige randomisierte Untersuchungen zum Einfluss humaner Milch auf die NEK-Inzidenz und die Mortalität Frühgeborener. Frauenmilch versus Formulanahrung wurde in mehreren Studien verglichen [10].

Zusammengefasst erhöht Formulanahrung die NEK-Inzidenz signifikant (RR 2,77; 95\% Cl 1,4-5,5). Es scheint auch ein Zusammenhang zwischen der in den ersten 14 Tagen gefütterten Frauenmilchmenge und dem späteren Risiko für NEK oder Tod zu bestehen. Der Anteil der Frauenmilch an der Gesamteinfuhr korreliert mit dem Überleben ohne NEK ( $\triangleright$ Abb.1). Die Wahrscheinlichkeit, eine NEK oder einen Tod zu erleiden, wurde in dieser Studie für jede 10-Prozent-Steigerung des Anteils an Frauenmilch um den Faktor 0,83 gesenkt [11].

Ob die Pasteurisierung von Frauenmilch deren protektiven Effekt auf die NEK mindert, ist unklar. Die in pasteurisierter Milch nachgewiesenen Veränderungen $(\triangleright$ Tab. 3) lassen dies aber vermuten.

\section{Probiotika}

Probiotika reduzieren bei sehr kleinen Frühgeborenen sowohl die NEK-Inzidenz als auch die Mortalität. Bis heute wurden in über 45 randomisierten Studien mehr als 9000 Frühgeborene untersucht. Die Studien unter- 


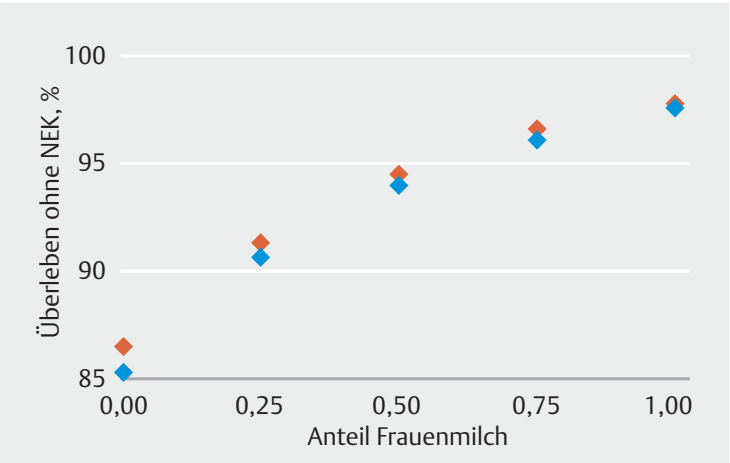

- Abb. 1 Überleben ohne NEK in Abhängigkeit vom Anteil der Frauenmilch an der Gesamtzufuhr innerhalb der ersten 14 Lebenstage. Rot: Tag 90; blau: Tag 114. Adjustiert für Geburtsgewicht, SGA, Ethnie, PDA, Lungenreife-Induktion, Beatmungsdauer und Klinik. Untersucht wurden 1272 Frühgeborene mit einem Geburtsgewicht von $401-1000 \mathrm{~g}$ (Daten aus [11]).

scheiden sich in verwendeten Präparaten, Dosis und methodischer Qualität. In einer 2017 publizierten Metaanalyse haben sich Chang und Mitarbeiter [12] auf Studien nach dem Jahr 2000 und auf solche mit einer ausreichend hohen Qualität (Jadad-Score 3-5) beschränkt. Die Vermutung aus früheren Metaanalysen, die Kombination mehrerer probiotischer Keime sei wirksamer als Monopräparate, hat sich klar bestätigt ( $>$ Tab.4).

\section{Empfehlung und Situation in Deutschland}

Viele Neonatologen konnten sich nur sehr schwer vorstellen, Frühgeborenen mit möglicherweise kompromittiertem Immunsystem vermehrungsfähige Keime
- Tab. 3 Folgen der Pasteurisierung humaner Milch.

\begin{tabular}{|l|l|}
\hline Milchkomponente & Pasteurisierung \\
\hline zelluläre Bestandteile & abgebaut \\
\hline Lactoferrin & mindestens halbiert \\
\hline Immunglobuline (sIgA, IgG) & halbiert \\
\hline Antioxidanzien & vermindert \\
\hline lösliches CD14 & minus 88\% \\
\hline Oligosaccharide & unverändert \\
\hline Amylase & minus 15\% \\
\hline Lipase & nicht nachweisbar \\
\hline LC PUFA & variabel \\
\hline Zytokine (IL-6, IL-10 etc.) & vermindert \\
\hline $\begin{array}{l}\text { Wachstumsfaktoren (EGF, } \\
\text { TGF- } \beta 1, \text { TGF- } \beta 2, \text { IGF) }\end{array}$ & variabel \\
\hline Hormone (Insulin, Adiponektin, & vermindert \\
\hline Erythropoetin) & wasserlöslich: vermin- \\
\hline Vitamine & dert; fettlöslich: stabil \\
\hline
\end{tabular}

zu verabreichen. Da die Metaanalysen einen erheblichen Effekt auf die NEK-Inzidenz und Mortalität nahelegten und insbesondere keine Nebenwirkungen berichtet wurden, begann 2007 das erste Perinatalzentrum mit der routinemäßigen Prophylaxe bei Frühgeborenen unter $1500 \mathrm{~g}$. Seitdem schlossen sich viele Level-I-Zentren dieser Routine an, so dass 2015 rund $84 \%$ der Kinder unter $1500 \mathrm{~g}$ in Deutschland Probiotika erhielten ( $\mathbf{A b b} . \mathbf{2}$ ).

- Tab.4 Metaanalyse randomisierter, kontrollierter Studien der Jahre 2001 - 2016 mit den Bedingungen Beginn der Probiotikagabe innerhalb der ersten 7 Lebenstage, Dauer mindestens 14 Tage sowie Gestationsalter $\leq 34$ Wochen und/oder Gewicht $\leq 1500 \mathrm{~g}$ [12].

\begin{tabular}{|c|c|c|c|c|c|c|c|}
\hline & \multirow{2}{*}{$\begin{array}{l}\text { Studien } \\
\text { N }\end{array}$} & \multicolumn{2}{|c|}{ Probiotika } & \multicolumn{2}{|c|}{ Kontrolle } & \multirow[t]{2}{*}{ Odds Ratio } & \multirow[t]{2}{*}{ Cl $95 \%$} \\
\hline & & NEK & Total & NEK & Total & & \\
\hline \multicolumn{8}{|l|}{ NEK } \\
\hline Kombinationspräparate & 11 & 35 & 1439 & 94 & 1450 & 0,36 & $0,24-0,53$ \\
\hline Lactobacillus sp. & 5 & 25 & 820 & 41 & 826 & 0,60 & $0,36-1,00$ \\
\hline Bifidobacterium sp. & 6 & 65 & 1130 & 76 & 1114 & 0,85 & $0,61-1,20$ \\
\hline Saccharomyces sp. & 3 & 18 & 290 & 20 & 276 & 0,80 & $0,41-1,56$ \\
\hline \multicolumn{8}{|l|}{ Tod } \\
\hline Kombinationspräparate & 10 & 75 & 1428 & 121 & 1439 & 0,58 & $0,43-0,79$ \\
\hline Lactobacillus sp. & 3 & 12 & 349 & 13 & 352 & 0,94 & $0,42-2,13$ \\
\hline Bifidobacterium sp. & 6 & 70 & 1130 & 74 & 1114 & 0,95 & $0,68-1,33$ \\
\hline Saccharomyces sp. & 2 & 9 & 239 & 10 & 240 & 0,90 & $0,36-2,26$ \\
\hline
\end{tabular}




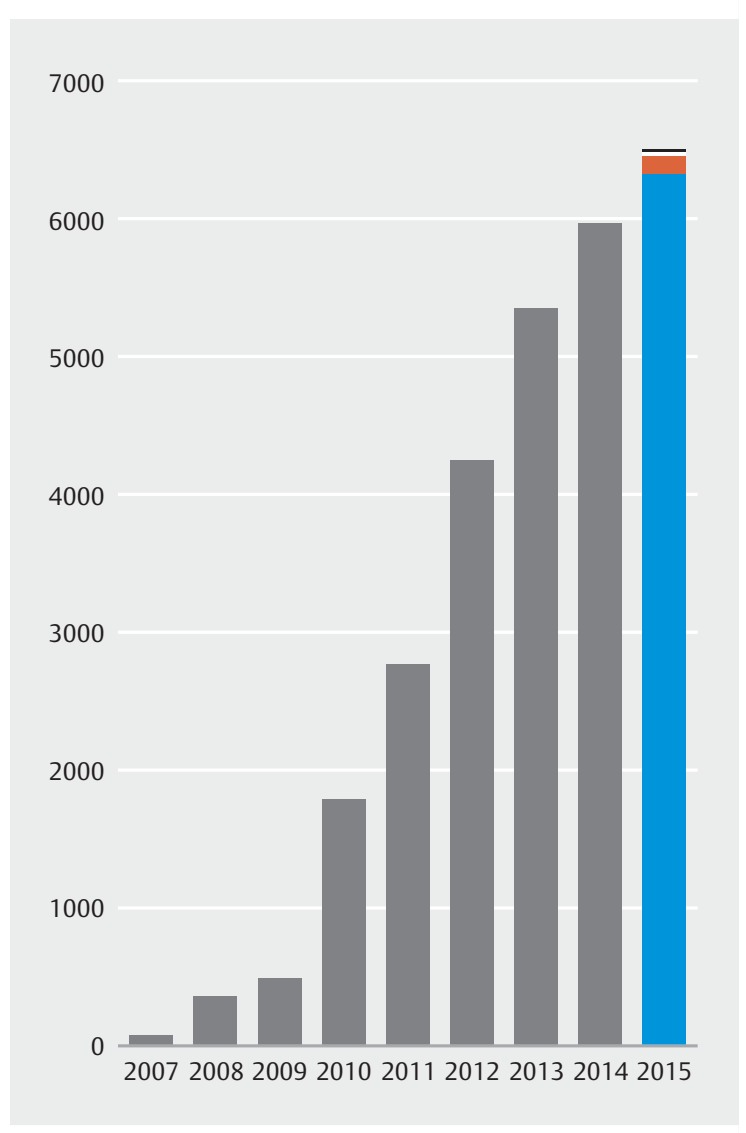

- Abb. 2 Probiotika in deutschen Level-I-Perinatalzentren. Anzahl der VLBW-Kinder, die in einem Level I-Zentrum, das routinemäßig Probiotika einsetzt, behandelt werden. Bei der Berechnung der Zahl der Probiotikaexponierten Kinder wurde die VLBW Fallzahl der einzelnen Zentren von 2014 zugrunde gelegt. Ergebnis einer Befragung von 162 Perinatalzentren mit einem Rücklauf von $97,5 \%$. blau - L. acidophilus und B. infantis; rotLactobacillus reuteri; weiß/schwarz-Kombination aus $>3$ Probiotika

In den meisten Studien wurden VLBW-Frühgeborene eingeschlossen. Täglich $1 \times 10^{9}$ CFU Lactobacillus acidophilus und $1 \times 10^{9}$ Bifidobacterium sp. ist die am besten untersuchte Probiotika-Kombination. In den randomisierten Studien wurden Probiotika überwiegend ab dem 3. Lebenstag und bis zu einem Alter von 6 Wochen, oder, falls früher, bis zur Entlassung verabreicht. In einer Kohortenstudie (1224 VLBW-Frühgeborene aus 3 Zentren) senkte auch eine zehntägige Probiotikagabe das NEK-Risiko signifikant, nicht aber die Mortalität [13].

In Deutschland sind verschiedene Präparate, die Lactobacillus acidophilus und Bifidobacterium infantum enthalten, erhältlich ( $>$ Tab.5).
- Tab. 5 Probiotikagabe bei Frühgeborenen.

\begin{tabular}{|c|c|}
\hline Frage & Antwort \\
\hline Wer? & $\begin{array}{l}\text { Frühgeborene }<1500 \mathrm{~g} \text { bei Geburt oder } \\
\text { einem Gestationsalter }<32 \text { Wochen }\end{array}$ \\
\hline Wer nicht? & $\begin{array}{l}\text { Kinder nach Abdominalchirurgie } \\
\text { (Einzelfallentscheidung; ggf. höheres } \\
\text { Risiko für Bakteriämie bei Stase/Pen- } \\
\text { delperistaltik) }\end{array}$ \\
\hline Wann? & wenn $\geq 2 \mathrm{ml} /$ Mahlzeit \\
\hline Was? & $\begin{array}{l}\text { täglich } 10^{9} \text { CFU Lactobacillus acidophi- } \\
\text { lus und } 10^{9} \text { CFU Bifidobacterium infan- } \\
\text { tis }\end{array}$ \\
\hline Handelsname? & z. B. Lactopia probio infant, Infloran \\
\hline Dauer? & $\begin{array}{l}\text { bis zum 42. Lebenstag oder bis Ent- } \\
\text { lassung (kumuliert ca. } 80 \times 10^{9} \mathrm{CFU} \text { ) }\end{array}$ \\
\hline
\end{tabular}

\section{Diagnose}

Die frühzeitige Diagnose der NEK ist schwierig, weil die Symptome unspezifisch sind und ein spezifischer Laborparameter (Marker für intestinale Schädigung) für die Routine nicht zur Verfügung steht.

\section{Klassische Definition nach Walsh und Kliegman (1986)}

Die Einteilung der NEK anhand diagnostischer Kriterien wurde erstmals von Bell und Mitarbeitern 1978 nach Auswertung der Verläufe von 48 betroffenen Kindern vorgeschlagen. Die Stadieneinteilung sollte eine Hilfe bei der Indikation zur Operation und für deren besten Zeitpunkt sein. Heute werden die Kriterien der Stadieneinteilung irrtümlicherweise auch zur Diagnose der NEK herangezogen. Die Bell-Klassifikation wurde 1986 von Walsh und Kliegman überarbeitet ( $\triangleright$ Tab.6).

Der Radiologe Arthur Steinen hat 1951 den Zusammenhang von NEK und Pneumatosis intestinalis beschrieben. Nicht selten dauert es viele Stunden, bis durch den radiologischen Nachweis intramuraler Luft die Diagnose gesichert werden kann. Häufig sind zu diesem Zeitpunkt die betroffenen Frühgeborenen bereits in einem sehr schlechten Zustand. Es gibt aber auch foudroyante Verläufe, bei denen sich die Klinik bei weitgehend unauffälligem Labor und anscheinend ohne Prodromi binnen weniger Stunden verschlechtert und die Diagnose einer NEK erst im OP gestellt bzw. die Operabilität nicht mehr erreicht wird. Nicht selten ist bei solchen Verläufen nahezu der gesamte (Dünn-) Darm betroffen (Enterocolitis necroticans totalis) und die Prognose besonders schlecht. Möglicherweise liegen dem foudroyanten Verlauf genetische Ursachen zugrunde. Die klinischen, biochemischen und radiologischen Zeichen lassen sich nicht immer exakt den von Walsh und Kliegman definierten Stadien zuordnen. 
- Tab.6 Klassifikation der NEK nach Walsh und Kliegman. Quelle: Gesellschaft für Neonatologie und Pädiatrische Intensivmedizin. Nekrotisierende Enterokolitis (NEK). AWMF-Leitlinien-Register Nr. 024/009).

\begin{tabular}{|c|c|c|c|}
\hline Stadium & Systemische Zeichen & Gastrointestinale Zeichen & Radiologische Zeichen \\
\hline IA (Verdachtsdiagnose) & \multirow[t]{3}{*}{$\begin{array}{l}\text { Temperaturinstabilität, Apnoen, } \\
\text { Bradykardien, Lethargie }\end{array}$} & $\begin{array}{l}\text { Magenreste, okkulte rektale Blu- } \\
\text { tungen, Erbrechen, geringgradige } \\
\text { abdominelle Distension }\end{array}$ & \multirow[t]{2}{*}{$\begin{array}{l}\text { normal oder geringe Dilatation, } \\
\text { geringgradiger lleus }\end{array}$} \\
\hline IB (Verdachtsdiagnose) & & blutige Stühle & \\
\hline $\begin{array}{l}\text { IIA (NEK gesichert, wenig } \\
\text { krank) }\end{array}$ & & $\begin{array}{l}\text { zusätzlich fehlende Darmgeräusche, } \\
\text { eindeutige abdominale Schmerzen }\end{array}$ & $\begin{array}{l}\text { zusätzlich Pneumatosis intesti- } \\
\text { nalis, mittel- bis hochgradiger } \\
\text { lleus mit dilatierten, stehenden } \\
\text { Darmschlingen }\end{array}$ \\
\hline IIB (mäßig krank) & $\begin{array}{l}\text { zusätzlich milde metabolische } \\
\text { Azidose, mäßige Thrombozyto- } \\
\text { penie }\end{array}$ & $\begin{array}{l}\text { zusätzlich Druckschmerz, evtl. } \\
\text { Resistenz im rechten Unterbauch, } \\
\text { evtl. Erythem der Bauchwand }\end{array}$ & $\begin{array}{l}\text { zusätzlich portalvenöses Gas, } \\
\text { evtl. Aszites }\end{array}$ \\
\hline $\begin{array}{l}\text { IIIA (fortgeschrittene NEK, } \\
\text { schwer krank, Darm intakt) }\end{array}$ & \multirow{2}{*}{$\begin{array}{l}\text { zusätzlich metabolische und respi- } \\
\text { ratorische Azidose, Neutropenie, } \\
\text { Sepsis mit Hypotension, Schock, } \\
\text { Bradykardie und DIC }\end{array}$} & \multirow{2}{*}{$\begin{array}{l}\text { zusätzlich generalisierte Peritonitis, } \\
\text { hochgradige abdominelle Distension } \\
\text { und Verfärbung, Resistenz im rech- } \\
\text { ten Unterbauch }\end{array}$} & zusätzlich Aszites \\
\hline IIIB (Darm perforiert) & & & zusätzlich Pneumoperitoneum \\
\hline
\end{tabular}

\section{HINTERGRUND}

\section{Pneumatosis intestinalis}

Vermutlich werden Paneth-Körnerzellen, die am Grund der Dünndarmkrypten in enger Nachbarschaft zu Arteriolen und Venolen liegen, durch bakterielle Toxine geschädigt. PanethZellen exprimieren auf der luminalen Seite Lipopolysaccharidrezeptoren und Toll-like-Rezeptoren und sezernieren Lysozyme, Peptidasen, Laktoferrin und Defensine. Sie sind Bestandteil der lokalen Immunabwehr und spielen eine wichtige Rolle bei der Pathogenese des Morbus Crohn. Mit der Zerstörung der Paneth-Zellen gelangen intrazelluläre Zytokine in das Darmlumen und von basolateral in die benachbarten Blutgefäße. Die Zytokine führen zu einer Inflammation und Schädigung des Endothels mit Aktivierung von Neutrophilen und Thrombozyten. Folgen der Fibrinablagerung und Plättchenaggregation sind Gerinnungsstörung, Vasokonstriktion und Mikrothromben, die bis zur Nekrose der Mukosa führen können. Spätestens jetzt können Bakterien durch die zerstörten Krypten die Submukosa erreichen und, sofern gasbildend, die pathognomonischen Blasen bilden.

Die korrekte und frühzeitige Diagnose der NEK ist aus mehreren Gründen erstrebenswert: für unsere Patienten soll eine Über- genauso wie eine Untertherapie vermieden werden; für Studienzwecke soll eine Kontamination mit falsch-positiven wie auch falsch-negativen Diagnosen vermieden werden; die NEK-Rate ist nicht zuletzt ein wichtiger Parameter der (vergleichenden) Qualitätssicherung.

Die Definition der NEK-Stadien nach Walsh und Kliegman stammt aus der Vor-Surfactant-Ära. Im Gegensatz zu den 1980er Jahren des letzten Jahrhunderts ist heute die NEK bei Frühgeborenen mit einem Geburtsge- wicht über $1500 \mathrm{~g}$ oder einer Schwangerschaftsdauer über 32 SSW selten. Bei diesen Frühgeborenen oder reifen Neugeborenen liegen der NEK meist andere Erkrankungen wie schwere intrauterine Wachstumsrestriktion, angeborene Herzfehler, Polyglobulie, schwere Diarrhö oder Sepsis zugrunde.

\section{Stadium I}

Zum Stadium I ( $\triangleright$ Tab.6) gehören milde systemische Zeichen wie Apnoe, Bradykardie oder Temperaturinstabilität. Milde intestinale Symptome wie geblähtes Abdomen, Magenreste und auch blutige Stühle können bei jeder Verschlechterung eines sehr kleinen Frühgeborenen auch ohne primär gastrointestinale Ursache beobachtet werden.

Die radiologischen Befunde sind unauffällig oder unspezifisch und können eine drohende NEK nicht von einem sog. CPAP-Bauch unterscheiden ( $\triangleright$ Abb. 3 ). Blutige Stühle werden nur bei einem von fünf NEK-Patienten unterhalb der 27.SSW beobachtet, weil die Blutungsquelle häufig proximal der lleozäkalklappe liegt. Bei einem Reifealter von 33-34 SSW werden dagegen bei jedem zweiten NEK-Patienten blutige Stühle beobachtet.

Andere Symptome, wie geblähtes Abdomen, Ileus oder Pneumoperitoneum sind dagegen häufiger bei sehr unreifen Frühgeborenen und werden mit zunehmendem Gestationsalter seltener beobachtet. Das Fehlen der für das Stadium I aufgezählten Merkmale schließt eine NEK also keineswegs aus [14]. Die Inkonsistenz der beschriebenen Symptome deutet darauf hin, dass sich verschiedene Krankheiten hinter Stadium I verbergen, die der klassischen NEK mehr oder weniger ähneln. 


\section{Merke}

Die Merkmale des Stadiums I sind unspezifisch und begründen die Diagnose NEK nicht.

\section{Stadium II (gesicherte NEK)}

Stadium II unterscheidet sich von Stadium I im Wesentlichen durch den radiologischen Nachweis intramuraler Luft (Pneumatosis intestinalis) oder Luft in der Pfortader. Der Nachweis intramuraler Luft ist häufig unsicher. Auch unter erfahrenen Radiologen ist die Interund Intraobserver-Reliabilität schwach. Im Gegensatz dazu lässt sich Luft in der Pfortader sonografisch einfach nachweisen. Ob Luft in der Pfortader ein prognostisch schlechtes Zeichen im Sinne einer NEK IIB ist, ist fraglich.

In einer Metaanalyse wurden Studien mit über 700 Frühgeborenen mit gesicherter NEK oder NEK-Verdacht analysiert [15]. Der sonografische Nachweis von Luft in der Pfortader hatte keine Bedeutung für die Prognose des weiteren Krankheitsverlaufs hinsichtlich Notwendigkeit einer Operation oder Mortalität. Dies ist umso erstaunlicher, als es sich bei dem Nachweis von Luft in der Pfortader um ein wesentliches Kennzeichen der fortgeschrittenen NEK nach Walsh und Kliegman handelt. Eine Erklärung könnte sein, dass dieser Befund heute häufiger sonografisch erhoben wird, als es früher radiologisch möglich war. Vielleicht kommt inm deshalb nicht mehr die schwerwiegende Bedeutung zu, die ihm Walsh und Kliegman für das Stadium IIB im Sinne einer Progredienz zumaßen.

Welche Rolle Artefakte beim Nachweis von intramuraler oder intravasaler Luft spielen, wurde nicht untersucht. Der Vergleich verschiedener sonografischer Zeichen bei Frühgeborenen mit NEK $(n=748)$ zeigt, dass Pneumatosis hepatis oder intestinalis sowie vermehrte Perfusion des Darmes und einfacher Aszites nicht signifikant mit Operation oder Tod korrelierten [15]. - Abb. 4 zeigt das relative Risiko verschiedener sonografischer Zeichen für das Outcome „NEK-Operation“ oder „Tod“.

\section{Stadium III}

Stadium III beschreibt schwer(st)kranke Patienten mit noch intaktem (IIIA) oder perforiertem Darm (IIIB) und Peritonitis. Der Übergang zum Multiorganversagen ist fließend. Es gibt keine befriedigende Definition der NEK sehr unreifer Frühgeborener. Die wichtigsten gastrointestinalen Differenzialdiagnosen sind in $>$ Tab. 7 dargestellt.

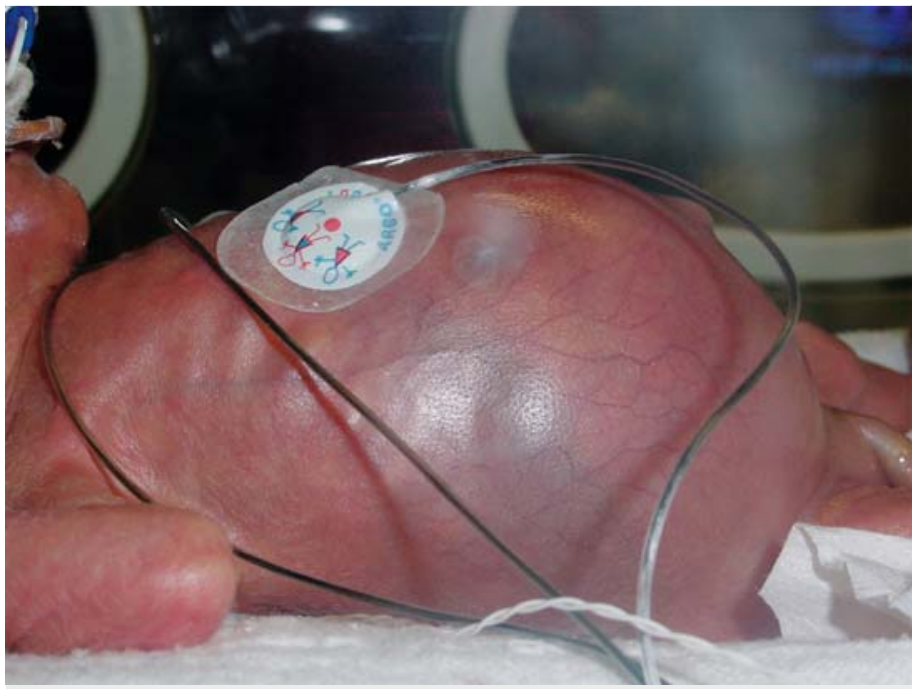

Abb. 3 Klinischer Befund einer NEK IA. Ausladendes Abdomen, prallelastische Bauchdecken, vermehrte Venenzeichnung, mäßig glänzende Haut. Nach wiederholter Entlastung von oral und rektal mit Magensonde bzw. Darmrohr und Reduktion des PEEP völlige Normalisierung des Befunds. Auch im weiteren Verlauf kein Anhalt mehr für eine NEK.

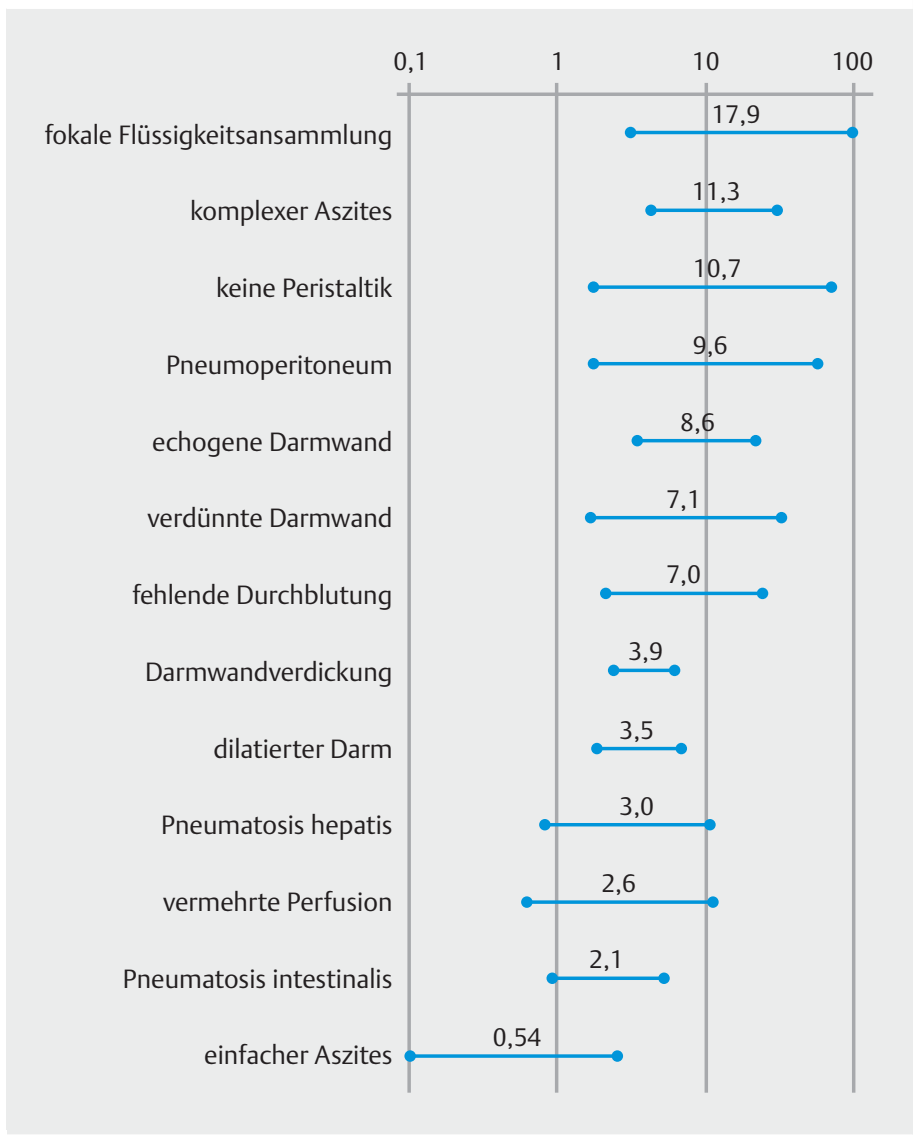

- Abb.4 Relatives Risiko für das Outcome „NEK-Operation“ oder „Tod“ für verschiedene sonografische Befunde. Die Balken bezeichnen das 95\%-Konfidenzintervall (Daten aus [15]). 
- Tab.7 Differenzialdiagnosen der nekrotisierenden Enterokolitis [21].

\begin{tabular}{|c|c|c|c|c|c|c|}
\hline & $\begin{array}{l}\text { Nahrungsun- } \\
\text { verträglichkeit }\end{array}$ & $\begin{array}{l}\text { „Preterm“ } \\
\text { NEK }\end{array}$ & Virale Enteritis & $\begin{array}{l}\text { Fokale intestinale } \\
\text { Perforation }\end{array}$ & $\begin{array}{l}\text { Ischämische } \\
\text { NEK }\end{array}$ & $\begin{array}{l}\text { Kuhmilchpro- } \\
\text { tein-Intoleranz }\end{array}$ \\
\hline $\begin{array}{l}\text { typisches Ge- } \\
\text { burtsgewicht (g) }\end{array}$ & $<1250$ & $<1250$ & & $<1000$ & $>1250$ & $>2000$ \\
\hline Alter (Wochen) & $<2$ & $>2$ & $>2$ & $<2^{1}$ & $<4$ & $>6$ \\
\hline Pneumatosis & nein & ja & $\begin{array}{l}\text { häufig < } 1250 \mathrm{~g} \text {, } \\
\text { selten > } 1250 \mathrm{~g}\end{array}$ & nein & ja & möglich \\
\hline $\begin{array}{l}\text { Pneumoperito- } \\
\text { neum }\end{array}$ & nein & $20-30 \%$ & $\begin{array}{l}30-40 \%<1250 \mathrm{~g}, \\
\text { ungewöhnlich } \\
>1250\end{array}$ & $100 \%$ & gelegentlich & selten \\
\hline blutige Stühle & nein & ungewöhnlich & ja & nein & ja & ja \\
\hline Ileus & nein & ja & $\begin{array}{l}\text { nicht zu Beginn } \\
\text { nicht }>1250 \mathrm{~g}\end{array}$ & variabel & ja & nein \\
\hline $\begin{array}{l}\text { Nahrungsmenge } \\
(\mathrm{ml} / \mathrm{kg} \times \mathrm{d})\end{array}$ & $<80$ & $>80$ & 120 & $<40$ & $>80$ & Kuhmilchprotein \\
\hline Häufung & nein & nein & ja & nein & nein & nein \\
\hline $\begin{array}{l}\text { Gerinnungs- } \\
\text { störung }\end{array}$ & nein & $\begin{array}{l}\text { abhängig vom } \\
\text { Schweregrad }\end{array}$ & $\begin{array}{l}\text { abhängig vom } \\
\text { Schweregrad }\end{array}$ & nein & $\begin{array}{l}\text { abhängig vom } \\
\text { Schweregrad }\end{array}$ & nein \\
\hline Keimnachweis & nein & & $\begin{array}{l}\text { Stuhl: Rota-, Astro- } \\
\text { Picorna-Virus }{ }^{2}\end{array}$ & unspezifisch & unspezifisch & nein \\
\hline OP-Situs & & $\begin{array}{l}\text { Pneumatosis mit } \\
\text { Mukosanekrose }\end{array}$ & Aszites, Nekrose & $\begin{array}{l}\text { fokale Perforation } \\
\text { Ileum oder Jejunum }\end{array}$ & $\begin{array}{l}\text { Pneumatosis mit } \\
\text { Mukosanekrose }\end{array}$ & Aszites, Nekrose \\
\hline
\end{tabular}

\section{Merke}

Der Beginn einer NEK ist von unspezifischen Symptomen gekennzeichnet. Die Abgrenzung von Differenzialdiagnosen ist häufig schwierig. Der röntgenologische oder sonografische Nachweis intramuraler Luft (Pneumatosis intestinalis) oder portalvenösen Gases begründet die „Arbeitsdiagnose“ NEK. Blutige Stühle sind kein Beweis für eine NEK.

\section{Laborparameter}

\section{I-FABP}

Das intestinale fettsäurebindende Protein (I-FABP) wird ganz überwiegend im Intestinum exprimiert, aufgrund seiner geringen Größe von 14kD früh bei einer Zellschädigung freigesetzt und renal eliminiert. Die Spezifität des I-FABP, gemessen im Serum oder Urin, erwies sich in verschiedenen klinischen Studien als hoch $(0,91)$, die Sensitivität betrug 0,64. Da der Test nicht kommerziell erhältlich ist, steht im Gegensatz zu vielen anderen Organen für den Darm kein spezifischer Marker einer Zellschädigung zur Verfügung [16].

\section{Thrombozyten}

Die Thrombozytenzahl ist zur Diagnose einer NEK nicht geeignet, aber ein wichtiger prognostischer Parameter. Der zu erwartende Schweregrad einer NEK verhält sich umgekehrt zum Nadir der Thrombozytenzahl. Eine Thrombozytopenie zu Beginn der NEK oder ein rascher Abfall der Thrombozytenzahl unter $100 / \mathrm{nl}$ ist ein prognostisch schlechtes Zeichen. Werte $>100 / \mathrm{nl}$ oder eine Normalisierung der Thrombozytenzahl binnen 24 Stunden sind selten mit einer Operation oder Tod assoziiert [17].

\section{C-reaktives Protein ( $\mathrm{CrP})$}

Das CrP ist ein Akute-Phase-Protein, das in der Signalkaskade nach Interleukin-6 und anderen Zytokinen erscheint. Die Expression des CrP in der Leber wird am stärksten durch Interleukin-6 angeregt und ist ca. 24 Stunden nach Erkrankungsbeginn messbar erhöht. Diese Latenz erklärt Fallberichte von foudroyanten Verläufen einer nekrotisierenden Enterocolitis totalis, bei denen das CrP unmittelbar präoperativ noch unauffällig war. Die Bestimmung des CrP hilft nicht bei der Unterscheidung zwischen NEK und anderen entzündlichen Erkrankungen. Als Verlaufsparameter ist das $\mathrm{CrP}$ wegen seiner Halbwertszeit von 12-36 Stunden bei Frühgeborenen gut geeignet [17]. 


\section{Monozytenzahl}

Die Infiltration von Makrophagen in das entzündliche Gewebe ist ein typisches Merkmal der NEK. Da sich die Makrophagen auch aus den Monozyten des Blutes rekrutieren, könnte ein Abfall der Monozytenzahl die NEK von anderen Ursachen des akuten Abdomens oder der Nahrungsunverträglichkeit unterscheiden. Es gibt Hinweise aus einer kleinen Studie, dass ein Abfall der absoluten Monozytenzahl gegenüber dem letzten verfügbaren Blutbild eine Sensitivität und Spezifität von 70 bzw. $71 \%$ aufweist. Als Cut-off war ein Abfall von $20 \%$ oder mehr definiert $[17,18]$.

Interleukin-6 (II-6)

IL-6 hat eine Schlüsselrolle bei der angeborenen Immunantwort. Hauptsächlich aus aktivierten Monozyten/Makrophagen gelangt IL-6 nach Kontakt mit bakteriellen, viralen oder Pilz-Antigenen in das Blut. Erhöhte IL-6-Konzentrationen findet man bei allen Entzündungsreaktionen. Das zirkulierende IL-6 wird von Leber und Nieren ausgeschieden, die Halbwertszeit im Serum liegt im Minutenbereich. IL- 6 ist deshalb als Verlaufsparameter ungeeignet. Vermutlich korreliert die IL-6Konzentration mit der Schwere und dem Ausgang der NEK. In einer kleinen Studie waren die präoperativen IL-6-Konzentrationen der Kinder mit letalem Ausgang signifikant höher als die der überlebenden (15327 [4480-27456] vs. 37 [23-455] ng/l) [19].

\section{Calprotectin}

Calprotectin wird in Neutrophilen exprimiert. Sein Nachweis im Stuhl weist auf eine intestinale Infiltration mit Neutrophilen hin. Der diagnostische Wert von Calprotectin im Stuhl wurde in mehreren Studien mit 601 Frühgeborenen untersucht. Calprotectin und NEK sind assoziiert, allerdings unterscheidet sich der Cut-off für Calprotectin von Studie zu Studie. Die Sensitivität variiert von $76-100 \%$, die Spezifität von 39-96\%. Eine abschließende Bewertung ist noch nicht möglich [17].

\section{Merke}

Bei Erkrankungsbeginn ist IL-6 der empfindlichste Parameter. CrP eignet sich zur Verlaufsbeurteilung. Die Thrombozytenzahl korreliert negativ mit der Schwere der NEK. Der darmspezifische Parameter I-FABP ist im Routinelabor zurzeit nicht verfügbar.

\section{Praktisches Vorgehen bei Verdacht auf NEK}

Die Therapie der NEK ist symptomatisch. In der Regel wird die enterale Nahrungszufuhr bereits bei Verdacht auf NEK beendet und ggf. mit einer breiten antibiotischen Therapie begonnen. Eine dicklumige Magenablaufsonde, Schmerztherapie und der Ausgleich von Elektrolytverschiebungen, Hypo- und Hyperglykämien sind selbstverständlich. Für die Therapie einer Sepsis sei auf die AWMF-Leitlinie und deren abteilungsspezifische Modifikationen verwiesen.

Der Nutzen antianaerober Antibiotika ist umstritten, für eine Nutzen-Risiko-Bewertung reichen die Daten nicht aus. Die Dauer der antibiotischen Therapie richtet sich nach dem Krankheitsverlauf. In der Regel kann die antibiotische Therapie bei einem $\mathrm{CrP}<10 \mathrm{mg} / \mathrm{l}$ beendet werden. Die Überlegenheit „runder“ Therapiedauern wie 5, 7, 10 oder 14 Tage gegenüber anderen ist ein Mythos. Bei einem stagnierenden CrP-Verlauf, der durch den Lokalbefund nicht erklärt werden kann, muss an eine Kathetersepsis gedacht werden, welche ohne Entfernung oder ggf. einen Wechsel des Katheters nicht therapiert werden kann. Dies gilt insbesondere bei Anzeichen für einen erhöhten Thrombozytenverbrauch.

Bei dringendem Verdacht auf NEK müssen eine (weitere) Dehnung der Darmwand und Verminderung der intestinalen Durchblutung verhindert werden. Um den intraabdominellen Druck möglichst gering zu halten, wird man eine nichtinvasive Ventilation frühzeitig durch eine endotracheale Intubation und maschinelle Beatmung ersetzen.

Pentoxifyllin ist ein Phosphodiesterase-Inhibitor, der die TNFa-Produktion hemmt. Es gibt Daten aus Tierversuchen und aus der klinischen Forschung, die zumindest bei der Sepsis eine Verbesserung der Hämodynamik und Hemmung der Entzündung durch Pentoxifyllin zeigen. Die randomisierten Studien bei Frühgeborenen mit Sepsis und/oder NEK sind von mittelmäßiger Qualität, so dass die in einer Metaanalyse zusammengefassten Effekte nur geringe Aussagekraft haben. Pentoxifyllin senkte die Mortalität bei Neugeborenen mit Sepsis ( $n=416$; $R R=0,57 ; 95 \% C l=0,35-0,93)$, Daten zur NEK liegen nicht vor [20].

Es empfiehlt sich, die Kinderchirurgie bereits bei Verdacht auf ein akutes Abdomen zu informieren. Die gemeinsame, wiederholte Beurteilung des klinischen Verlaufs und die Diskussion der Befunde erleichtern die Entscheidung für oder gegen eine chirurgische Intervention ( $\mathbf{A} \mathbf{b} \mathbf{b} \mathbf{b} \mathbf{5})$. Die Bedeutung anderer radiologischer Zeichen zur Beurteilung des Schweregrads ist in

- Abb. 4 dargestellt. 


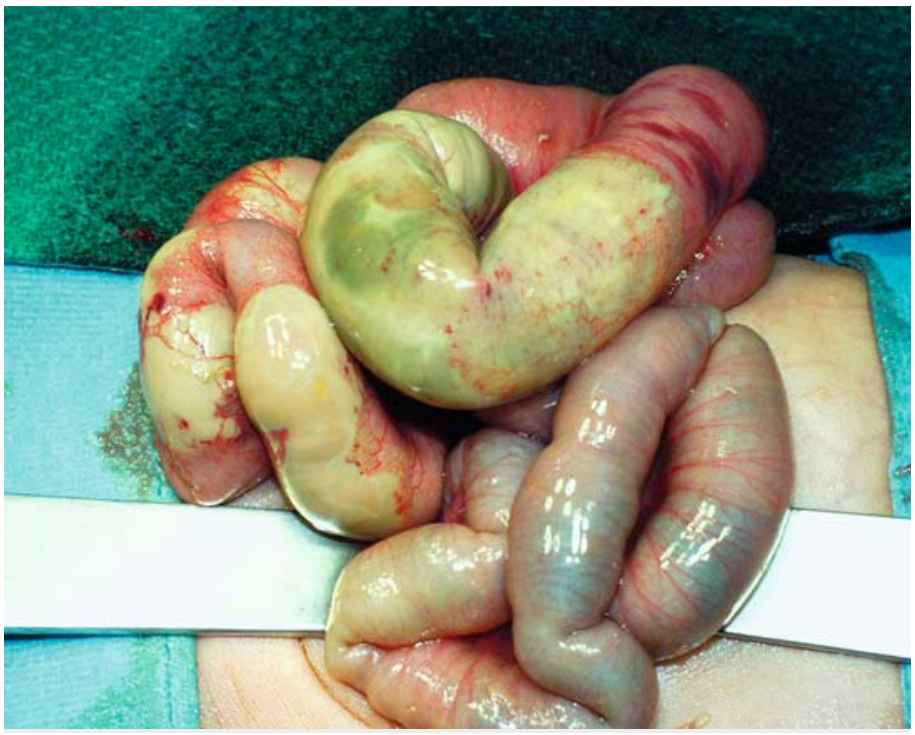

Abb.5 Operationssitus einer nekrotisierenden Enterokolitis. Überwiegend minderperfundiertes Intestinum mit ausgedehnten nekrotischen Arealen.

\section{Chirurgische Therapie}

Die Operationsindikation wird individuell und in Abhängigkeit vom klinischen Verlauf, der Bildgebung und dem Labor gestellt. Das Bauchwanderythem ist Zeichen einer klinischen Verschlechterung und gilt insbesondere bei gleichzeitiger Azidose und Hypotension als relative Operationsindikation. Eine mittels Pneumoperitoneum nachgewiesene Perforation gilt als absolute OP-Indikation.

Ziel der Operation ist die Entlastung des Darmes durch ein Stoma oder mehrere Stomata, die Verbesserung der Perfusion durch Druckentlastung und die Entfernung nekrotischer Anteile. Das Vorgehen kann sich zwischen den Kliniken deutlich unterscheiden. In Europa und den USA ist die Laparotomie mit zwei von drei Operationen der häufigste Eingriff. Vorteile sind die Entfernung nekrotischen Gewebes und die Entlastung des Darmes.

\section{FALLBEISPIEL}

Verlegung eines reifen, eutrophen Knaben zur Hypothermietherapie bei hypoxisch-ischämischer Enzephalopathie nach Schulterdystokie. Abbruch der Hypothermie nach 50 Stunden wegen klinischer Verschlechterung mit Hypotonie, ausladendem Abdomen und Nahrungsunverträglichkeit. $\mathrm{CrP} 38 \mathrm{mg} / \mathrm{l}$, Thrombozyten in $24 \mathrm{~h}$ von $155 / \mathrm{nl}$ auf $56 / \mathrm{nl}$ gefallen; Monozyten von $0,8 / \mathrm{nl}$ auf $0,04 / \mathrm{nl}$ gefallen; Röntgen ( $>$ Abb. 6 ): Ileus mit Pneumatosis intestinalis. Katecholaminbedarf. Laparotomie 9 Stunden nach Beginn der Symptome wegen Pneumoperitoneum (s.u.): Resektion des Colon descendens und sigmoideum, Anlage eines Ileostoma. Nachweis von Candida albicans und Clostridium perfringens in der Bauchhöhle. Bis zum endgültigen Verschluss der Bauchdecke 6 Spülungen bei temporärem Bauchdeckenverschluss. (Teil-) Parenterale Ernährung für 4 Monate. Reanastomose und Fortsetzung des Nahrungsaufbaus, Entlassung im Alter von 5 Monaten.

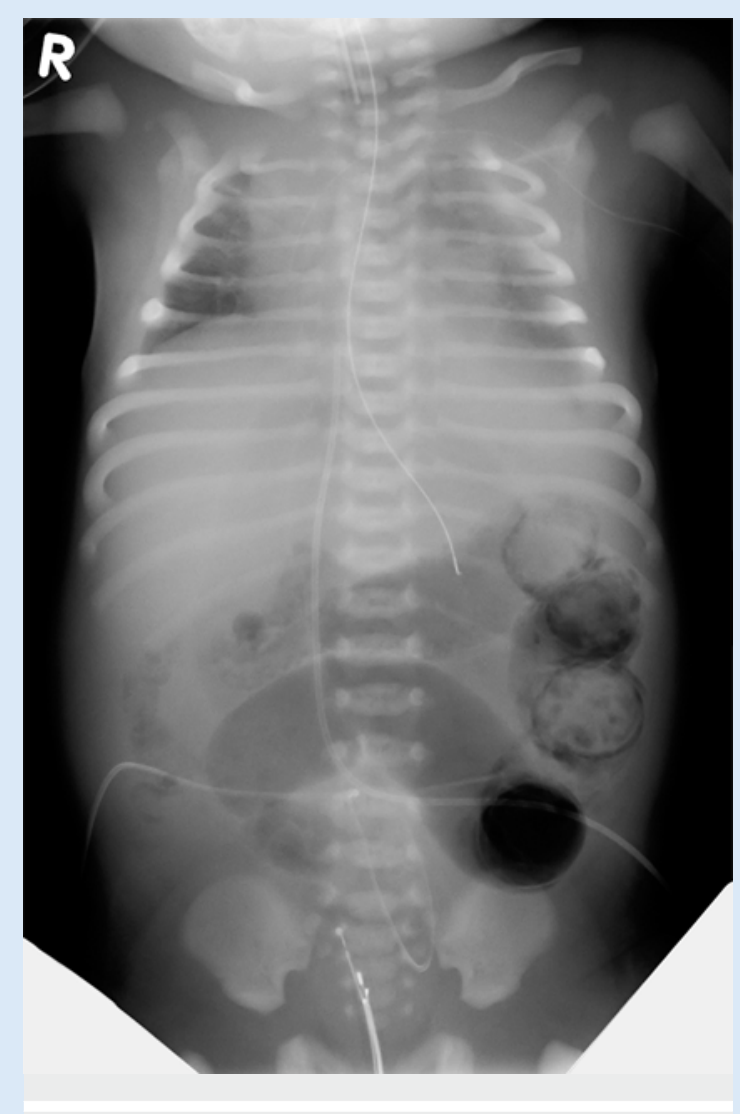

- Abb. 6 Röntgenuntersuchung von Thorax und Abdomen. Die Aufnahme wurde 7 Stunden nach Symptombeginn und 2 Stunden vor der ersten Laparotomie angefertigt. (Quelle: Kinderradiologie AUF DER BULT) 
Die Laparotomie ist aufwendiger als die Peritonealdrainage, und bei der Reanastomosierung kommt es meist zu weiterem Verlust von Darm. Die Peritonealdrainage kann eine sinnvolle Option für nichtoperable Patienten sein. In ca. ein Sechstel der Fälle reicht sie als alleinige Maßnahme aus, bei einem Sechstel der Fälle folgt später eine Laparotomie, die wegen der Verwachsungen im Vergleich zum primären Eingriff technisch schwieriger sein kann.

Bei ausgedehnten Nekrosen oder weiterer klinischer Verschlechterung werden nicht selten Relaparotomien nötig. Um den abdominellen Druck möglichst gering zu halten, kann es erforderlich sein, das Abdomen offen zu lassen und nur steril abzudecken.

Insbesondere nach der Anlage eines hohen Enterostomas können sich der enterale Nahrungsaufbau und die Stomapflege sehr schwierig gestalten. Ob das Umfüllen des Chymus vom oralen in das aborale Stoma den Nahrungsaufbau und das Gedeihen beschleunigt, ist noch unklar. Die mittel- und langfristigen potenziellen Folgen einer NEK-OP wurden eingangs beschrieben.

\section{KERNAUSSAGEN}

- Die nekrotisierende Enterokolitis (NEK) ist wegen ihrer hohen Mortalität und Langzeitmorbidität gefürchtet.

- Die Ätiologie der NEK ist multifaktoriell und noch nicht genügend verstanden.

- Wichtigstes Merkmal der betroffenen Kinder ist die Unreife. Besonderheiten des naiven Immunsystems, verminderte Toleranz gegenüber hypoxisch-ischämischem Stress und ein verändertes Mikrobiom sind Substrate der Unreife. Genetische Faktoren werden diskutiert.

- Die Diagnose der NEK und die Abgrenzung gegenüber anderen, vor allem septischen Krankheitsbildern, ist schwierig. Ein spezifischer Laborparameter oder ein spezifisches radiologisches Zeichen ist nicht bekannt.

- Die Therapie der NEK ist symptomatisch und nicht selten chirurgisch.

- Besondere Bedeutung kommt der NEK-Prävention zu: Lungenreife-Induktion, Ernährung mit humaner Milch und eine Kombination aus mindestens zwei Probiotika senken das NEK-Risiko signifikant.

\section{Schlüsselwörter}

Nekrotisierende Enterokolitis, Pneumatosis, Probiotika, Lungenreife, Spendermilch, Thrombozytopenie
Interessenkonflikt

Reisekosten und Vortragshonorare der Firmen Nestlé und Milupa.

\section{Autorinnen/Autoren}

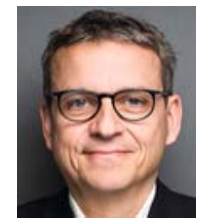

\section{Florian Guthmann}

Jahrgang 1963, Prof. Dr. med. Nach Studium und Promotion an der Freien Universität Berlin Ausbildung zum Kinderarzt, SP Neonatologie und Habilitation an der Charité. Dort stellvertr. Klinikdirektor. Weiterbildung zum Master of

Medical Education an der Universität Heidelberg. Seit 2012 Chefarzt am Kinder- und Jugendkrankenhaus AUF DER BULT in Hannover.

\section{Korrespondenzadresse}

Prof. Dr. med. Florian Guthmann, MME

Allgemeine Kinderheilkunde Neonatologie

AUF DER BULT

Kinder- und Jugendkrankenhaus

Janusz-Korczak-Allee 12

30173 Hannover

E-Mail: guthmann@hka.de

Wissenschaftlich verantwortlich gemäß

Zertifizierungsbestimmungen

Wissenschaftlich verantwortlich gemäß Zertifizierungsbestimmungen für diesen Beitrag ist Prof. Dr. med. Florian Guthmann

Literatur

[1] Patel RM, Kandefer S, Walsh MC et al. Causes and timing of death in extremely premature infants from 2000 through 2011. N Engl J Med 2015; 372: 331 - 340

[2] Zani A, Pierro A. Necrotizing enterocolitis: controversies and challenges. F1000 Research 2015; 4: (F1000 Faculty Rev) 1373

[3] Yee WH, Soraisham AS, Shah VS et al. Incidence and timing of presentation of necrotizing enterocolitis in preterm infants. Pediatrics 2012; 129: e298-304

[4] Goulet O, Baglin-Gobet S, Talbotec C et al. Outcome and long-term growth after extensive small bowel resection in the neonatal period: a survey of 87 children. Eur J Pediatr Surg 2005; 15: $95-101$

[5] Diamanti A, Basso MS, Castro M et al. Irreversible intestinal failure: prevalence and prognostic factors. J Pediatr Gastroenterol Nutr 2008; 47: 450-457

[6] Duro D, Mitchell PD, Kalish LA et al. Risk factors for parenteral nutrition-associated liver disease following surgical therapy for necrotizing enterocolitis: A Glaser Pediatric Research Network Study [corrected]. J Pediatr Gastroenterol Nutr 2011; 52: 595-600 
[7] Rees CM, Pierro A, Eaton S. Neurodevelopmental outcomes of neonates with medically and surgically treated necrotizing enterocolitis. Arch Dis Child Fetal Neonatal Ed 2007; 92: F193-198

[8] Samuels N, van de Graaf RA, de Jonge RC] et al. Risk factors for necrotizing enterocolitis in neonates: a systematic review of prognostic studies. BMC Pediatr 2017; 17: 105 [doi: 10.1186/s12887-017-0847-3]

[9] Roberts D, Brown J, Medley N et al. Antenatal corticosteroids for accelerating fetal lung maturation for women at risk of preterm birth. Cochrane Database Syst Rev 2017; 3: CD004454

[10] Quigley M, McGuire W. Formula versus donor breast milk for feeding preterm or low birth weight infants. Cochrane Database Syst Rev 2014; 4: CD002971

[11] Meinzen-Derr J, Poindexter B, Wrage L et al. Role of human milk in extremely low birth weight infants' risk of necrotizing enterocolitis or death. J Perinatol 2009; 29: 57-62

[12] Chang HY, Chen JH, Chang JH et al. Multiple strains probiotics appear to be the most effective probiotics in the prevention of necrotizing enterocolitis and mortality: An updated meta-analysis. PLoS One 2017; 9; 12: e0171579

[13] Guthmann F, Arlettaz Mieth R, Bucher HU et al. Short courses of dual-strain probiotics appear to be effective in reducing necrotising enterocolitis. Acta Paediatrica 2016; 105 : $255-259$

[14] Sharma R, Hudak ML, Tepas JJ 3rd et al. Impact of gestational age on the clinical presentation and surgical outcome of necrotizing enterocolitis. J Perinatol 2006; 26: 342 - 347

[15] Cuna AC, Reddy N, Robinson AL et al. Bowel ultrasound for predicting surgical management of necrotizing enterocolitis: a systematic review and meta-analysis. Pediatr Radiol 2017: doi:10.1007/s00247-017-4056-x
[16] Cheng S, Yu J, Zhou M et al. Serologic Intestinal-Fatty Acid Binding Protein in Necrotizing Enterocolitis Diagnosis: A Meta-Analysis. Biomed Res Int 2015; 2015: 156704

[17] Gephart SM, Gordon PV, Penn AH et al. Changing the paradigm of defining, detecting, and diagnosing NEC: Perspectives on Bell's stages and biomarkers for NEC. Sem Ped Surg 2018; $27: 3-10$

[18] Remon J, Kampanatkosol R, Kaul RR et al. Acute drop in blood monocyte count differentiates NEC from other causes of feeding intolerance. J Perinatol 2014; 34: 549 - 554

[19] Bhatia AM, Stoll BJ, Cismowski MJ et al. Cytokine levels in the preterm infant with neonatal intestinal injury. Am J Perinatol 2014; 31: 489-496

[20] Pammi M, Haque KN. Pentoxifylline for treatment of sepsis and necrotizing enterocolitis in neonates. Cochrane Database Syst Rev 2015; 3: CD004205

[21] Gordon PV, Swanson JR, Attridge JT et al. Emerging trends in acquired neonatal intestinal disease: is it time to abandon Bell's criteria? J Perinatol 2007; 27: 661-671

Bibliografie

DOI https://doi.org/10.1055/s-0042-110454

Neonatologie Scan 2018; 07: 121-134

(c) Georg Thieme Verlag KG Stuttgart · New York ISSN 2194-5462 


\section{Punkte sammeln auf CME.thieme.de}

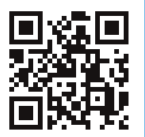

Diese Fortbildungseinheit ist 12 Monate online für die Teilnahme verfügbar. Sollten Sie Fragen zur Online-Teilnahme haben, finden Sie unter http://cme.thieme.de/hilfe eine ausführliche Anleitung. Wir wünschen viel Erfolg beim Beantworten der Fragen!

Unter https://eref.thieme.de/ZZWHDPR oder über den QR-Code kommen Sie direkt zum Artikel zur Eingabe der Antworten.

VNR 2760512018154652333

\section{Frage 1}

Ein Frühgeborenes, 26+4/7 SSW, enteral ernährt, CPAP mit $\mathrm{FiO}_{2} 0,21$, jetzt 2 Wochen alt, „gefällt der betreuenden Schwester nicht“. Das Abdomen ist ausladend, im Vergleich zu gestern mehr Apnoen, Bradykardien, Sättigungsabfälle. Sie nehmen Blut ab und röntgen das Abdomen. Welche Symptomkombination spricht 2 Stunden später am ehesten für das Vorliegen einer NEK?

A blutige, dünne Stühle, $\mathrm{CrP} 46 \mathrm{mg} / \mathrm{l}$

B Ileus und druckdolentes Abdomen, Thrombozyten 80/nl

C Aszites rechter Unterbauch, CrP $46 \mathrm{mg} / \mathrm{l}$

D Leukozyten 15,6/ $\mu$ l, dilatierte Darmschlingen (Röntgen)

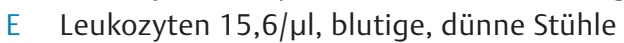

\section{Frage 2}

Welche Faktorenkonstellation in der Anamnese (siehe Frage 1) hätte das Risiko für das Auftreten einer NEK am meisten erhöht?

A Ernährung mit Frühgeborenen-Nahrung (Formula) antibiotische Behandlung der Mutter für 5 Tage bis zur Geburt - Gewicht unter der 3. Perzentile

B antibiotische Behandlung der Mutter für 5 Tage bis zur Geburt - Gewicht unter der 3. Perzentile - Ernährung mit Muttermilch

C Gewicht unter der 3. Perzentile - Ernährung mit Muttermilch - Zwillingsschwangerschaft

D Ernährung mit Muttermilch - Zwillingsschwangerschaft männliches Geschlecht

E Zwillingsschwangerschaft - männliches Geschlecht Ernährung mit Frühgeborenen-Nahrung (Formula)

\section{Frage 3}

Welche Aussage zur Therapie der NEK trifft zu?

A Bei gesicherter NEK Stadium II wird eine antibiotische Therapie für 10 Tage empfohlen.

B Ein Antibiotikum, das anaerobe Keime erfasst, ist zwingend nötig.

C Die Indikation zur Operation wird nach evidenzbasierten Kriterien gestellt.

D Die Kinderchirurgie soll frühzeitig in die Überlegungen einbezogen werden.

E Pentoxifyllin ist fester Bestandteil der NEK-Therapie.

\section{Frage 4}

Sie werden zu einem Frühgeborenen (28 SSW, Geburtsgewicht $1260 \mathrm{~g})$ gerufen, das durch Temperaturinstabilität, Magenreste, Apnoen, Bradykardien, Lethargie, abdominelle Distension und blutige Stühle auffällt. Heute, am 12. Lebenstag, erhält es Muttermilch mit Fortifier. Welche Aussage ist richtig?

A Da der Zustand nicht besorgniserregend ist, verordnen Sie Nahrungskarenz für zwei Mahlzeiten und CrP-Kontrolle zum Ausschluss einer Infektion.

B Sie diagnostizieren eine NEK und ordnen Nahrungskarenz und eine breite antibiotische Therapie, auch gegen Anaerobier, an. Sie informieren die Kinderchirurgie.

C Sie sind hinsichtlich der Diagnose unsicher und veranlassen Röntgen, II-6, CrP, Blutbild, Stuhl auf Viren und ordnen Nahrungskarenz an.

D Sie sind hinsichtlich der Diagnose unsicher, ordnen Nahrungskarenz sowie Magensonde auf Ablauf an und warten auf die Oberärztin, die in 1 h 45 min zum Frühdienst kommt.

E Sie sind hinsichtlich der Diagnose NEK sicher. Da Sie aber sonografisch keinen Anhalt für Luft in der Darmwand oder Leber finden, ändern Sie Ihre Diagnose auf Sepsis. 


\section{Punkte sammeln auf CME.thieme.de}

Fortsetzung ...

\section{Frage 5}

Ein Kollege ruft Sie zu einem Kind in reduziertem Allgemeinzustand. Geburt nach 35 SSW mit $2480 \mathrm{~g}$, jetzt am errechneten Termin und $3190 \mathrm{~g}$ schwer. Wegen der in den letzten 2 Stunden mehrfach abgesetzten dünnen Stühle mit Blutbeimengung hat er eine NEK diagnostiziert. Welche weiteren Informationen unterstützen die Diagnose?

A Pneumatosis intestinalis - gestern Umstellung von Muttermilch auf Pre-Nahrung

B Mutter heute wegen Infekt zu Hause - verminderter Turgor

C Lactatazidose - rezidivierendes Erbrechen

D AV-Kanal (komplett) - Hämatokrit 0,69l/I

E gestern ähnliches Bild bei anderem Kind im selben Zimmer - Aszites

\section{Frage 6}

Welche Maßnahmen senken die NEK-Inzidenz und die Mortalität Frühgeborener?
A Flüssigkeitsrestriktion und spätes Abnabeln
$B$ orale Antibiotika und Lungenreife-Induktion
C Lungenreife-Induktion und Probiotika
D Probiotika und Muttermilch
E Muttermilch und Flüssigkeitsrestriktion

\section{Frage 7}

Welche Antwort beschreibt die Aussagekraft verschiedener Laborparameter für Diagnose und Prognose der NEK am besten?

A CrP: hohe Sensitivität; Interleukin-6: Verlaufsparameter

B Interleukin-6: Verlaufsparameter; Thrombozyten: Schwere der Erkrankung

C Thrombozyten: Schwere der Erkrankung; I-FABP: hohe Spezifität

D I-FABP: niedrige Spezifität; CrP: hohe Spezifität

E CrP: hohe Spezifität; Interleukin-6: hohe Sensitivität

\section{Frage 8}

Probiotika wurden in zahlreichen randomisierten Studien mit Frühgeborenen getestet. Welche Aussage trifft zu?

A Probiotika erhöhen die Sepsisrate (Blutkultur positiv).

B Bei Frühgeborenen unter 26 SSW ist kein Effekt zu erwarten.

C Escherichia coli (Nissle), Lactobacillus sp., Saccharomyces sp., Bifidobacterium sp. sind gleichermaßen geeignet.

D In den meisten Studien wurde das Probiotikum ab Tag 3 für 6 Wochen verabreicht.

E Kombinationspräparate haben keinen Vorteil gegenüber Monopräparaten.

\section{Frage 9}

Welche Aussage zu Prognose und Folgen der NEK trifft nicht zu?

A Rund $20 \%$ der überlebenden NEK-Patienten entwickeln ein Kurzdarmsyndrom.

B Die neurologische Entwicklung ist nach OP signifikant schlechter als nach konservativer Therapie.

C Die Letalität der NEK (Stadium 2 oder höher) liegt bei $15-30 \%$.

D Ein Jejunostoma erschwert den enteralen Nahrungsaufbau und erfordert meist eine langwierige (teil-)parenterale Ernährung.

E Die Dünndarmtransplantation ist etablierte Therapie nach ausgedehnter Darmresektion.

\section{Frage 10}

Die Ernährung Frühgeborener beeinflusst die Inzidenz der NEK. Welche Aussage trifft zu?

A Lange parenterale Ernährung reduziert die Inzidenz der NEK.

B Ernährung mit Muttermilch senkt die NEK-Inzidenz auf $1 / 3$ bis $1 / 4$ im Vergleich zu Formula.

C Keine oder nur langsame $(10 \mathrm{ml} / \mathrm{kg}$ tägl.) Nahrungssteigerung reduziert die Inzidenz der NEK.

D Früher enteraler Nahrungsbeginn erhöht das Risiko für eine NEK.

E Die tägliche Steigerung der enteralen Ernährung um $30-35 \mathrm{ml} / \mathrm{kg}$ steigert das NEK-Risiko. 